\title{
PRINCIPAL BUNDLES ON ELLIPTIC FIBRATIONS *
}

\author{
RON Y. DONAGI ${ }^{\dagger}$
}

\begin{abstract}
A central role in recent investigations of the duality of F-theory and heterotic strings is played by the moduli of principal bundles, with various structure groups $G$, over an elliptically fibered Calabi-Yau manifold (on the heterotic side), and specifically over an elliptic-K3-fibered CalabiYau, on the F-theory side. In this note we propose a simple algebro-geometric technique for studying the moduli spaces of principal G-bundles on an arbitrary variety $\mathrm{X}$ which is elliptically fibered over a base S: The moduli space itself is naturally fibered over a weighted projective base parametrizing spectral covers $\tilde{S}$ of $\mathrm{S}$, and the fibers are identified as translates of distinguished Pryms of these covers. In nice situations, the generic Prym fiber is isogenous to the product of a finite group and an abelian subvariety of $\operatorname{Pic}(\tilde{S})$.
\end{abstract}

1. Introduction. Moduli spaces of principal $G$-bundles on $\mathrm{K} 3$ surfaces, and on Calabi-Yaus of other dimensions, are basic ingredients of the compactification of heterotic strings, especially for structure groups $E 8 \times E 8$ and $S O(32)$. More recently, compactifications of $F$-theory [1] have suggested the special importance of moduli spaces of $G$-bundles on elliptically fibered Calabi-Yaus, with $G$ a semisimple subgroup of $E 8 \times E 8$. The purpose of this note is to propose a general technique for studying $G$ bundles on (not necessarily CY) elliptic fibrations $X \rightarrow S$, with arbitrary semisimple structure group $G$.

The idea is quite simple. To a principal $G$-bundle $P$ over $X$ we associate its spectral data. This consists of the cameral cover $\tilde{S} \rightarrow S$, a Galois cover with covering group the Weyl group $W$ of $G$; of a collection of multisection maps $v_{\lambda}: \tilde{S} \rightarrow X$, one for each character $\lambda \in \Lambda$ of the maximal torus $T \subset G$, subject to the condition of $W$-equivariance (the spectral covers are the various images of $\tilde{S}$ in $X$ ); and of a $T$-bundle over $\tilde{S}$, subject to a certain twisted form of $W$-equivariance. When the cameral cover is reasonably nice, it determines the distinguished $\operatorname{Prym} \operatorname{Prym}(\tilde{S} / S)$, an extension of an abelian variety by a finite group. The $G$-bundles on $X$ with a given cameral cover $\tilde{S}$ (and any consistent collection of maps to $X$ ) can then be parametrized by the distinguished Prym. An open subset of the moduli of $G$-bundles can thus be fibered over the parameter space for nice cameral covers, the fibers being the distinguished Pryms. When the cameral cover is not-so-nice, the description becomes less precise; but the whole construction has a local character, so bad behavior can be traced to specific singular loci. We avoid much of the difficulty by considering regularized bundles, or bundles with some additional structure, specified by a reduction of the structure group, over each $s \in S$, to an abelian subgroup which is the centralizer in $G$ of a regular element. (We call such a subgroup a regular centralizer.) Nice bundles have a unique regularization, special ones may have large families of regularizations, and others will have none, but can become regularized after some blowing up in the base $S$.

The reason that regular centralizers are the right structure can be seen already from the behavior of $G$-bundles on a single elliptic curve $E$, and already for $G=S L(2)$. The structure group of "most" semistable $G$-bundles on $E$ can be reduced to a maximal

\footnotetext{
*Received February 6, 1997; accepted for publication May 16, 1997.

†Institute for Advanced Study, Princeton, NJ 08540, USA, and Department of Mathematics, University of Pennsylvania, Philadelphia, PA 19104, USA (donagi@IAS.EDU).
} 
torus $T \subset G$. For $G=S L(2)$, there is an essentially unique exception: the non-trivial extension of $\mathcal{O}$ by itself (or the same tensored with one of the four spin bundles). The structure group in this case can still be reduced to an abelian subgroup, though not to $T$ : it can be reduced to the group of upper triangular matrices with 1 or -1 on the diagonal, i.e. to the centralizer of a non-zero nilpotent element in $S L(2)$. As an abstract group, this is $\mathbf{Z}_{2}$ times the additive group $(\mathbf{C},+)$. Now the family of bundles which admit a reduction to a given torus $T$ is parametrized by $\mathbf{P}^{1}$, or more naturally by $E$ modulo its involution. There are only 8 isomorphism classes of bundles with reduction to a nilpotent regular centralizer: the above four extensions of $L$ by $L$, where $L$ is a spin bundle, as well as the four trivial extensions $L \oplus L$. An important point is that all regular centralizers in $G$ fit nicely into one family, $\mathcal{C}$. For $S L(2)$, the base of this family is $\mathbf{P}^{2}=\left(\mathbf{P}^{1} \times \mathbf{P}^{1}\right) / \mathbf{Z}_{2}$ : the tori are parametrized by the complement of the diagonal $\mathbf{P}^{1}$, while the exotics sit over $\mathbf{P}^{1}$. Over this $\mathbf{P}^{2}$ lives the space parametrizing $S L(2)$-bundles on $E$ together with a reduction of their structure group to some regular centralizer. This space looks like the threefold $\mathbf{P}^{1} \times \mathbf{P}^{1} \times E$ divided by the obvious $\mathbf{Z}_{2}$, with the singularities blown up (yielding fibers of type $I_{0}^{*}$ over $\mathbf{P}^{1}$ ) and the components of multiplicity 2 discarded. Over this threefold there is a "universal $\mathcal{C}$-bundle", from which our $G$-bundles can be induced. We will see that this picture generalizes to any $G$, and is the basic ingredient behind the reconstruction of a $G$-bundle from its spectral data.

Our construction is motivated by our previous work [2], and in a sense is contained in it. The objects studied in [2] may appear, at first sight, different than the ones that concern us here. There we were mostly interested in $K$-valued Higgs fields on $S$, where $K$ is a line (or vector) bundle on $S$. As we review in section 4 , the parametrization of these " $K$-valued" objects is reduced to that of abstract, or unvalued, objects, and those are precisely the ones that come up in connection with $G$-bundles on $X \rightarrow S$. From this point of view, we may think of a $G$-bundle on $X$ as a Higgs bundle on $S$ whose "values" are in the elliptic curves of the fibration.

The main construction, or rather its reduction to the construction in [2], is in section 4. Some basics on $G$-bundles on a single elliptic curve are gathered in section 2 , while the behavior in families is discussed in section 3 . There we also address the technical question of how to describe the family of all cameral covers. For this we need to understand the global properties of the moduli of $G$-bundles on a fixed elliptic curve, as well as the modular behavior seen when the curve is varied. The former were worked out by Looijenga [3] and Bernstein and Shvartsman [4], and the latter, for all simply connected groups except E8, by Wirthmuller [5].

It is a pleasure to thank Ed Witten, for asking the questions about moduli of $G$ bundles on elliptic fibrations which got me interested in the subject, and for drawing my attention to references [3], [4]. His joint work with Friedman and Morgan [6], just posted to hep-th, has some overlap with this note. Roughly speaking, the emphasis in [6] is on a description of the parameter space for spectral covers, while we focus on the fiber, which parametrizes bundles with a given cover. The theorem of Looijenga is recovered in full in [6], and there is also a discussion of Wirthmuller's work. Friedman, Morgan and Witten also obtain applications to the duality between F-theory and the heterotic string. They present several beautiful descriptions of the moduli space of $G$-bundles on an elliptic curve. While we use deformations (of semisimple and semistable bundles, in the beginning of section 2) only to obtain a rather rough local picture of this moduli space, they base their main construction on the deformations of a "minimally unstable" bundle. This gives them a global description, as well as the proof 
of Looijenga's theorem. In our approach, we take the results of Looijenga, BernsteinShvartsman, and Wirthmuller as given, and use them to describe the parameter space of the covers. The main novelty of our approach is the direct construction of the bundle corresponding to given spectral data, via the regular centralizers.

I am also grateful to Tony Pantev, for many discussions and for showing me an advance copy of another related work [7], to Eduard Looijenga who explained his works [3] to me and who told me about [5], and to Pierre Deligne for some very helpful comments on the manuscript. I have also benefitted greatly from conversations with J. Bernstein, R. Lazarsfeld, R. Livne, and V. Sadov on various aspects of the work described here. This work was partially supported by NSF grant DMS95-03249, a Lady Davis Fellowship from the Hebrew University, and grants from the University of Pennsylvania Research Foundation and the Harmon Duncombe Foundation.

2. G-bundles on elliptic curves. Let $G$ be a connected, simply-connected complex semisimple group, $T$ its maximal torus, $E$ an elliptic curve, i.e. a non singular curve of genus 1 with a marked point $0 \in E$. The moduli space $M_{E}^{T}$ of degree- 0 semistable $T$-bundles on $E$ is $\operatorname{Hom}(\Lambda, \check{E})$, where $\Lambda:=\operatorname{Hom}(\mathrm{T}, \mathbf{C})$ is the lattice of characters of $G$, and $\check{E}:=\mathrm{Pic}^{0} \mathrm{E}$ is the dual elliptic curve, which is naturally identified with $E$. This moduli space is an abelian variety, in fact it is (non-naturally) isomorphic to $E^{r}$, where $r$ is the rank of $G$. It comes with a natural action of the Weyl group $W$, as well as a natural polarization (cf.[3]) whose degree is the discriminant of $\Lambda$. Over $E \times M_{E}^{T}$ there is a universal $T$-bundle. In case $T=\mathbf{C}^{*}, M_{E}^{T}$ is just the dual elliptic curve $\check{E}$, and the universal bundle is the Poincare bundle. Via the unnatural identification of the general $M_{E}^{T}$ with $E^{r}$, the universal bundle becomes the sum of $r$ Poincare bundles pulled back from the $r$ factors. In this abelian situation there is actually a moduli space of all (not necessarily semistable) $T$-bundles: just replace $\check{E}=\mathrm{Pic}^{0} \mathrm{E}$ throughout by Pic E.

The moduli space $M_{E}^{G}$ of semistable $G$-bundles on $E$ is the quotient $M_{E}^{T} / W$, where the Weyl group $W$ acts through $\Lambda$. This moduli space parametrizes not isomorphism classes but $s$-equivalence classes of semistable $G$-bundles. There is an open set where the two notions coincide, but over smaller strata there will be a finite number of isomorphism classes represented by each point in moduli. The set of isomorphism classes maps naturally to $M_{E}^{G}$, and this map behaves nicely in families. (This classifying map is, of course, part of the definition of a coarse moduli space.) This map can be given as follows: any semistable principal $G$-bundle $P$ on $E$ has a semistable reduction of its structure group to the Borel subgroup $B \subset G$, in other words, it is associated to some semistable $B$-bundle $P_{B}$. The quotient map $B \rightarrow T$ then gives an associated $T$-bundle $P_{T}$ which, up to the action of $W$, is independent of the choice of Borel reduction. The existence of a Borel reduction can be seen, e.g., by finding a flat holomorphic connection on $P([8])$ and noting that the holonomy is abelian, as image of $\pi_{1}(E)$. (The entire discussion extends easily to non simply connected groups, as long as we restrict attention to those bundles which are liftable to the simply connected covering group.)

We can develop a feel for this moduli space by considering the deformation theory of semisimple bundles, i.e. ones associated to $T$-bundles. Let $P_{T}$ be a principal $T$ bundle on $E$, and $P$ the associated (semisimple) $G$-bundle. Deformations of $P$ are unobstructed, parametrized by $H^{0}(\operatorname{ad} P)^{*}$. Now

$$
\operatorname{ad}(P)=\operatorname{ad}\left(P_{T}\right) \oplus\left(\oplus_{\alpha} L_{\alpha}\right),
$$

where the sum is over the roots $\alpha \in R$ of $G$, and $L_{\alpha}$ is the line bundle associated 
to $P_{T}$ by the root $\alpha$. For generic $P$ each $L_{\alpha}$ is a non-trivial line bundle of degree 0 on $E$, so $H^{0}(\operatorname{ad} P)=H^{0}\left(\operatorname{ad} P_{T}\right)$. This means that all deformations of such generic $P$ come from deformations of $P_{T}$. But for non-generic semisimple $P$, there can be other deformations. The possible types of non-generic semisimple $P$ are indexed by subroot systems: An arbitrary $P_{T}$ is given by a homomorphism $p: \Lambda \rightarrow E$, which determines a sub root system $R^{\prime}:=R \cap \operatorname{ker}(\mathrm{p})$, generating a sublattice $\Lambda^{\prime} \subset \operatorname{ker}(\mathrm{p}) \subset \Lambda$. The structure group of this $P_{T}$ then reduces to $\bar{T}:=\operatorname{Hom}\left(\Lambda / \Lambda^{\prime}, \mathbf{C}^{*}\right) \subset \mathrm{T}$. Let $G^{\prime}$ be the centralizer $Z_{G}(\bar{T})$, with Lie algebra $\mathbf{g}^{\prime}$. The $\alpha$ for which $L_{\alpha}$ is trivial are precisely the roots of $\mathbf{g}^{\prime}$, so the deformations are now parametrized by the dual $\mathbf{g}^{\prime *}$, and the general deformation has structure group $G^{\prime}$. Since $G^{\prime}$ is the automorphism group of $P$, it acts on the deformation space, and the isomorphism type is fixed along $G^{\prime}$-orbits. The semisimple locus looks near $P_{T}$ like $\mathbf{t}$, and modulo $G^{\prime}$ this becomes $\mathbf{t} / W^{\prime}$ ( $\mathbf{t}$ is the Cartan subalgebra, $W^{\prime}$ is the Weyl group of $R^{\prime}$ ), while the new transversal deformations look like the nilpotent cone in $\mathbf{g}^{\prime}$. Modulo $G^{\prime}$, this gives just a finite set of types, with a non-separated topology. In case $G=S L(n)$ we recover Atiyah's description [9] of vector bundles on elliptic curves as sums of indecomposables. The possible root systems $R^{\prime}$ correspond in this case to faces of the Weyl chamber, or equivalently to parabolic subgroups containing $B$. For other groups there may be additional possibilites; e.g. for $G_{2}$ there are, in addition to the parabolic types $0, A_{1}^{\text {short }}, A_{1}^{\text {long }}$, and $G_{2}$, also the two possibilities $A_{2}^{\text {long }}$ and $A_{1}^{\text {long }} \times A_{1}^{\text {short }}$, which arise when the image of $p: \Lambda \rightarrow E$ happens to consist of points of order two. But in any case, the nilpotent cone breaks into a finite number of orbits. The closed orbit is 0 , corresponding to the semisimple bundles. At the opposite end, there is a unique dense orbit, corresponding to regular bundles. For such bundles $P, H^{0}(\operatorname{ad} P)$ is again of the smallest possible dimension, namely $r$. It is the centralizer in $\mathbf{g}^{\prime}$ of a regular nilpotent element.

There is a natural, "universal", space $\mathcal{U}_{E}$ parametrizing $G$-bundles on $E$ together with a trivialization (frame) over $0 \in E$ and a reduction to a regular centralizer. To describe it, we start with the quotient $G / T$ which parametrizes pairs $T^{\prime} \subset B^{\prime}$ consisting of a maximal torus $T^{\prime}$ and a Borel subgroup containing it, or equivalently a torus with a choice of chamber. The Weyl group $W$ acts on $G / T$. The quotient $G / N$ (where $N:=N_{G}(T)$ is the normalizer of $T$ in $G$ ) parametrizes tori $T^{\prime}$. Equivalently, $G / T$ and $G / N$ can be described in terms of Cartan subalgebras $\mathbf{t}^{\prime} \subset \mathbf{g}$ and Borels $\mathbf{b}^{\prime} \subset \mathbf{g}$. In [D] we introduced the parameter spaces $\overline{G / N}$ and $\overline{G / T}$, parametrizing all regular centralizers $C \subset G$, respectively pairs $C \subset B$. By definition, $\overline{G / N}$ is an open subset of the closure of $G / N$ in $\operatorname{Grass}(r, \mathbf{g})$. (The closure itself contains some abelian subalgebras which are not regular centralizers and therefore need to be discarded.)

Over $\overline{G / N}$ there is the vector bundle $\underline{\mathrm{c}} \rightarrow \overline{G / N}$ of rank $r$ abelian subalgebras of g, and the corresponding abelian group scheme $\mathcal{C} \rightarrow \overline{G / N}$ whose fibers are the regular centralizers in $G$. Let $\varpi$ be the projection $\overline{G / N} \times E \rightarrow \overline{G / N}$. Then $\varpi^{*} \mathcal{C}$ is an abelian group scheme over $\overline{G / N} \times E$. The cohomology sheaf $R^{1} \varpi_{*} \varpi^{*} \mathcal{C}$ can be represented by an analytic or algebraic space $u^{\prime}: \mathcal{U}_{E}^{\prime} \rightarrow \overline{G / N}$ whose fiber over $C \in \overline{G / N}$ is $H^{1}\left(E, C\left(\mathcal{O}_{E}\right)\right)$, the moduli space of $C$-bundles on $E$. We will usually restrict attention to the subfamily $u: \mathcal{U}_{E} \rightarrow \overline{G / N}$ parametrizing semistable $C$-bundles. Over $\mathcal{U}_{E}$ there is a universal principal $\mathcal{C}$-bundle (more precisely, a $u^{*} \mathcal{C}$-bundle) $P_{\mathcal{U}_{E}}^{C} \rightarrow \mathcal{U}_{E} \times E$. Since $u^{*} \mathcal{C}$ is a subgroup scheme of the trivial group scheme $G$ over $\mathcal{U}_{E} \times E$, we get an associated principal $G$-bundle $P_{\mathcal{U}_{E}} \rightarrow \mathcal{U}_{E} \times E$. The bundles $P_{\mathcal{U}_{E}}^{C}$ and $P_{\mathcal{U}_{E}}$ are uniquely characterized by the properties:

* The restriction of $P_{\mathcal{U}_{E}}^{C}$ to $\mathcal{U}_{E} \times\{0\}$ is the trivial $\mathcal{C}$-bundle. 
* the restriction of $P_{\mathcal{U}_{E}}^{C}$ to $\{x\} \times E$ (where $x:=(C, p) \in \mathcal{U}_{E}, C$ a regular centralizer, $p$ the isomorphism class of a $C$-bundle over $E$ ) is a $C$-bundle on $E$ in the class $p$, and the restriction of $P_{\mathcal{U}_{E}}$ is the associated $G$-bundle.

We could rephrase this as saying that $\mathcal{U}_{E}$ is a fine moduli space for the data it parametrizes: a $G$-bundle together with a trivialization over $0 \in E$ and a semistable reduction to a regular centralizer. The basic reason for existence of the universal family is that the objects parametrized have no automorphisms: we killed them by fixing the trivialization.

Over any one stratum of $\overline{G / N}$ it is easy to describe $\mathcal{U}_{E}$ and $P_{\mathcal{U}_{E}}^{C}$. For instance, over the open stratum $G / N$, we start with the Poincare $T$-bundle over $\mathcal{M}_{E}^{T} \times E$, cross this with $G / T$, and divide by $W$ which acts on both $G / T$ and $\mathcal{M}_{E}^{T}$.

A related object which we shall need is the quotient $\overline{\mathcal{U}}_{E}:=\left(\overline{G / T} \times \mathcal{M}_{E}^{T}\right) / W$. There is a natural morphism $f: \mathcal{U}_{E} \rightarrow \overline{\mathcal{U}}_{E}$ which, over each $C \in \overline{G / N}$, maps $H^{1}\left(E, C\left(\mathcal{O}_{E}\right)\right)$ to its compact part $H^{1}\left(E, C^{\prime}\left(\mathcal{O}_{E}\right)\right)$, where $C^{\prime}$ is the quotient of $C$ by its unipotent part, $C^{\prime}:=C /(C \cap[B, B])$ for a Borel $B \supset C$. Between fibers over points of the open stratum $G / N$, this map is surjective (in fact, an isomorphism); but over the whole base its image is the constructible set

$$
\overline{\mathcal{U}}_{E}^{\prime}:=\left\{(C, t) \in \overline{G / T} \times \mathcal{M}_{E}^{T} \mid \operatorname{Stab}_{W}(C) \subset \operatorname{Stab}_{W}(t)\right\} / W .
$$

As described in the introduction, for $G=S L(2)$ we have $\overline{G / T}=\mathbf{P}^{1} \times \mathbf{P}^{1}$ and $\overline{G / N}=\mathbf{P}^{2}$. The fiber of either $\mathcal{U}_{E}$ or $\overline{\mathcal{U}}_{E}{ }^{\prime}$ over a point not in the diagonal is $E$. Over points of the diagonal, the fibers in $\mathcal{U}_{E}, \overline{\mathcal{U}}_{E}{ }^{\prime}$ and $\overline{\mathcal{U}}_{E}$ respectively are: four lines, four points, and $\mathbf{P}^{1}$. We may think of $\overline{\mathcal{U}}_{E}{ }^{\prime}$ as parametrizing isomorphism classes of $G$-bundles having a semistable reduction to a regular centralizer $C$, together with a trivialization over $0 \in E$. The additional data in the fiber of $\mathcal{U}_{E}$ over $\overline{\mathcal{U}}_{E}$ chooses such a $C$-structure. Thus $f$ is the forgetful map sending a $C$-struture to its associated $G$-structure. The point is that the dimension of the normalizer $N_{G}(C)$ can be greater than $r$, so there can be a non-trivial family of $C$-bundles whose associated $G$-bundles are all isomorphic.

3. Families of moduli spaces. Let $\pi: X \rightarrow S$ be an elliptic fibration with a section, with non-singular $X, S$. We want to put the basic objects from the previous section into families parametrized by $S$. It will be convenient to work instead with a (singular) Weierstrass model $\bar{X} \rightarrow S$, given by an equation of the form $y^{2}=x^{3}+$ $b_{2} x+b_{3}$, where $b_{i}$ is a section of $L^{\otimes 2 i}$ and $L$ is $\pi_{*}$ of the relative canonical bundle $K_{\pi}$.

Since $\pi$ has a section, we can identify $\mathrm{Pic}^{0}$ of a smooth fiber $E_{s}:=\pi^{-1}(s)$ with $E_{s}$. Globally, the relative Jacobian $\operatorname{Pic}^{0}(\overline{\mathrm{X}} / \mathrm{S})$ is the complement in $\bar{X}$ of the locus of singular points of fibers. (By definition, this singular locus includes any fiber component of multiplicity $>1$.) The reason for this is that a section of $\pi: \bar{X} \rightarrow S$ must have intersection number 1 with each fiber, so it cannot pass through a singular point. This leads immediately to identification of $\mathcal{M}_{X / S}^{T}$ with the $r$-th cartesian power of $\bar{X}-\operatorname{Sing}(\bar{X})$ over the base $S$.

A satisfactory construction of $\mathcal{M}_{X / S}^{G}$ is somewhat more delicate. Looijenga shows [3] (see also [4]) that for simply connected group $G$ and for fixed, non-singular $E, \mathcal{M}_{E}^{G}$ is a weighted projective space. The weights are the Dynkin indices of the dual Dynkin diagram of $G$ (i.e. the coefficients of the highest short root when expressed as a sum of simple roots, plus the coefficient 1 for the affine root). In order to be able to describe the relative object $\mathcal{M}_{X / S}^{G}$, we need a way of relating nearby fibers. A general 
construction of a flat connection on such families was carried out by Saito [10] but more immediately useful results are in [5]. Wirthmuller takes the base $S$ to be a modular curve, so that Jacobi forms give sections of powers of the "theta" bundle (giving the polarization) on $\mathcal{M}_{X / S}^{T}$, and we are looking for the $W$-invariants among them. These are now bi-graded, by what he calls "weight" (as modular form) and index. The "indices" correspond to the weights in Looijenga's weighted projective space, and also to the power of the theta bundle in which the section lives. I will refer to Wirthmuller's "weights" as degrees, to avoid confusion with Looijenga's weights. These degrees correspond to powers of $L$, the Weierstrass line bundle. One of them always turns out to be 0 , the others are the degrees of the basic $G$-invariant polynomials. It is not clear a-priori why there should be a natural way to pair these two sequences, of indices and degrees. The end result is that $\mathcal{M}_{E}^{G}$, for simple $G$ other then $E 8$, can be identified with the quotient $\left(\oplus_{i=0}^{r} L^{d_{i}}\right) / \mathbf{C}^{*}$, where $d_{0}=0,\left\{d_{i}\right\}_{i=1}^{r}$ are the degrees of the invariant polynomials, and $\mathbf{C}^{*}$ acts with weights equal to the Dynkin indices for the dual Kac-Moody algebra.

At least for some groups, these results are elementary. For $S L(r+1)$, all indices equal 1 , while the degrees are $0,2,3,4, \ldots r+1$. So $\mathcal{M}_{E}^{G}$ is the ordinary projective space, projectivization of $\mathcal{O} \oplus L^{2} \oplus \ldots \oplus L^{r+1}$. The fact that $\mathcal{M}_{E}^{G}$ is a projective space follows directly from Abel-Jacobi: $\mathcal{M}_{E}^{T}$ is the locus of $(r+1)$-tuples of points in $E$ which add up to $0, W$ is the symmetric group, so the quotient is the variety of effective divisors in the linear system $r+1$ times the origin, a projective space of dimension $r$. But we can really identify this space: for $r=1$ it is the $\mathbf{P}^{1}$ with coordinate $\mathrm{x}$, of which the Weierstrass equation exhibits $E$ as a double cover. A basis of sections of $\mathcal{O}(1)$ is given by 1 (of degree 0 ) and $x$ (of degree 2). For $r=2$ we get the $\mathbf{P}^{2}$ with functions $1, x, y$, etc. Similar or easier arguments show that for $G$ either $S O(2 r+1)$ or $S p(r)$, the moduli space $\mathcal{M}_{E}^{G}$ is the $r$-th symmetric product of the basic $\mathbf{P}^{1}$, so it can be identified with the projectivization (all weights equal 1) of $\mathcal{O}+L^{2}+L^{4}+\ldots+L^{2 r}$. For $S p(r)$, this is as it should be: the Dynkin diagram of the Kac-Moody algebra $\left(\check{C}_{r}\right)=D_{r+1}^{(2)}$ has all indices equal to 1 . But the Dynkin diagram of the Kac-Moody algebra $\left(\check{B}_{r}\right)=A_{2 r-1}^{(2)}$ has its three extreme indices equal to 1 , while the others equal 2 , so we expect a weighted projective space of weights $(1,1,1,2, \ldots, 2)$. And that is exactly what we get, if we replace $S O(2 r+1)$ by the simply connected $\operatorname{Spin}(2 r+1)$ : the double covering group yields a 4 -sheeted covering moduli space (the elliptic curve has 4 two-torsion points), which can be identified as the unique $\mathbf{Z} / 2 \times \mathbf{Z} / 2$ cover each of whose three $\mathbf{Z} / 2$ quotients is branched along two of the three hyperplanes in $\mathbf{P}^{r}=\operatorname{Sym}^{r} \mathbf{P}^{1}$ corresponding to $r$-tuples containing one of the three roots of the Weierstrass equation.

I have not worked out the missing case of $E 8$, nor the remaining non simply connected groups. Presumably, even if $\mathcal{M}_{E}^{G}$ does not always turn out to be a weighted projective space, it can still be described by some universal construction in terms of the Weierstrass line bundle $L$. In particular, there is then a natural way to extend the family of quotients $\mathcal{M}_{E_{s}}^{T} / W$ to a locally trivial family over the entire base $S$ (including the discriminant) of any Weierstrass family. The most familiar instance of this is when $G=S L(2)$ : there the resulting $\mathcal{M}_{X / S}^{G}$ is the $\mathbf{P}^{1}$-bundle over $S$ obtained as projectivization of $\mathcal{O} \oplus L$, which is the quotient of the Weierstrass model by its natural involution fixing the 0 -section.

We also need to note that the spaces $\mathcal{U}_{E}$ and $\overline{\mathcal{U}}_{E}$ parametrizing $\mathcal{C}$ - and $G$-bundles likewise extend in families to form objects $\mathcal{U}_{X / S}$ and $\overline{\mathcal{U}}_{X / S}$ respectively. For $\overline{\mathcal{U}}_{X / S}$ we 
take $\left(\overline{G / T} \times \mathcal{M}_{X / S}^{T}\right) / W$. Near $s$ corresponding to non-singular fiber $E_{s}$, this looks like a bundle over $S$ with fibers $\overline{\mathcal{U}}_{E}$. At singular $s$, we have already removed the singularities of $\mathcal{M}_{X / S}^{T}$, so we get a non-singular, but somewhat smaller, object. Likewise, we restrict $\mathcal{U}_{X / S}$ to the open subset which maps to $\overline{\mathcal{U}}_{X / S}$. Its points then parametrize $\mathcal{C}$-bundles on the $E_{s}$, and there is again a universal $\mathcal{C}$-bundle on $\mathcal{U}_{X / S} \times_{S} X$. (It is possible that for those groups for which Wirthmuller-type constructions work we could extend these universal objects to some of the singular loci, but we have not pursued this, and the construction presented below seems to avoid the issue.)

4. Spectral parametrization of bundles. In this section we describe the equivalence between regularized $G$-bundles on an elliptic fibration with a section and the corresponding spectral data. We fix an elliptic fibration $\pi: X \rightarrow S$ with a "zero" section $\sigma: S \rightarrow X$, where $X, S$ are smooth. Let $E_{s}:=\pi^{-1}(s)$ denote a fiber. Given a principal $G$-bundle $P$ on $X$, let $P_{S}:=P_{\mid \sigma(S)}$ be the restriction to the zero section. There are associated bundles of groups $\mathrm{AdP}, \mathrm{AdP}_{\mathrm{S}}$ over $X, S$ respectively. The sheaf of automorphisms along the fibers $E_{s}, \operatorname{Aut}_{\mathrm{S}}(\mathrm{P}):=\pi_{*} \mathrm{AdP}$, can be identified as a subsheaf of $\mathrm{AdP}_{\mathrm{S}}$. There is also an associated bundle $\overline{P_{S} / N}$, whose fiber over $s \in S$ is the family of regular centralizers associated to the fiber $P_{s}$ over $\sigma(s)$.

A section $c: S \rightarrow \overline{P_{S} / N}$ determines an abelian group scheme $\mathcal{C} \rightarrow S$, a subgroup scheme of the group scheme $\mathrm{AdP}_{\mathrm{S}}$. A $\pi^{*} \mathcal{C}$-torser $P^{\mathcal{C}}$ on $X$ then induces a $G$-bundle $P=P^{\mathcal{C}} \times_{\mathcal{C}} G$. By a regularized $G$-bundle we mean such a triple $\{P, c: S \rightarrow$ $\left.\overline{P_{S} / N}, P \mathcal{C}\right\}$, or equivalently a reduction of the structure of $P$ to a group scheme $\mathcal{C}$ of regular centralizers. This group subscheme $\mathcal{C} \subset \operatorname{AdP}_{\mathrm{S}}$ is contained in $\operatorname{Aut}_{\mathrm{S}}(\mathrm{P})$, and extends naturally to a group subscheme of AdP. A key point is that an everywhere regular, semisimple and semistable bundle $P$ (i.e. one whose restriction $P_{s}$ to each $E_{s}$ has these properties) has a unique regularization, with $\mathcal{C}=\operatorname{Aut}_{\mathrm{S}}(\mathrm{P})$. But there are other regularized bundles, whose underlying $G$-bundles may not be everywhere, or even anywhere, regular. Yet these bundles too can be parametrized by their spectral data. (Being regularized means that we have chosen a reduction to a regular centralizer subgroup of the automorphisms along each $E_{s}$, not necessarily that those automorphisms form a regular centralizer themselves.) If $P$ is known to be regular, semisimple, and semistable only for generic $s \in S$ then a regularization is still unique if it exists; in general though, we may have to blow up the base $S$ to find a regularization.

A cameral cover of $S$ is a $W$-Galois cover $\tilde{S} \rightarrow S$ which is modelled on $\overline{G / T} \rightarrow$ $\overline{G / N}$. ("modelled on" means "obtained locally as pullback via maps of $S$ to the base".) Recall that $\overline{G / N}$ parametrizes regular centralizers in $G$, while $\overline{G / T}$ parametrizes pairs of a regular centralizer and a Borel containing it.

A regularized bundle $\left\{P, c, P^{\mathcal{C}}\right\}$ determines the following data:

(1) A cameral cover $\tilde{S} \rightarrow S$.

(2) A $W$-equivariant morphism $v: \tilde{S} \rightarrow \mathcal{M}_{X / S}^{T}$. (Equivalently, a morphism $v^{\prime}$ : $\Lambda \times \tilde{S} \rightarrow \mathrm{Pic}^{0}(\mathrm{X} / \mathrm{S})$. By way of terminology, we refer to the image of $v$ as the universal spectral cover. The various other spectral covers are the images under $v^{\prime}$ of $\lambda \times \tilde{S}$, for $\lambda \in \Lambda$.)

(3) A homomorphism $\ell: \Lambda \rightarrow$ Pic $\tilde{\mathrm{S}}$ (or equivalently, a $T$-bundle on $\tilde{S}$ ) satisfying the twisted $W$-equivariance property of [2].

These are obtained as follows.

(1) The cameral cover $\tilde{S}$ is the cover of $S$ induced via $c$ from the cover $\overline{P_{S} / T} \rightarrow \overline{P_{S} / N}$, which indeed looks locally like $\overline{G / T} \rightarrow \overline{G / N}$.

(2) A point $\tilde{s} \in \tilde{S}$ above $s \in S$ corresponds to a choice of Borel in $P_{s}$ containing the 
regular centralizer $\mathcal{C}_{s} \subset \operatorname{Aut}_{\mathrm{s}}\left(\mathrm{P}_{\mathrm{s}}\right)$. This extends uniquely to a subbundle of Borels in $P_{\mid E_{s}}$. Via the natural quotient map $B \rightarrow B /[B, B]=T$, there is an associated $T$-bundle, identified with a point of $\mathcal{M}_{E_{s}}^{T}$. When $\tilde{S}$ is reduced, we get the map $v$ by letting $s$ and $\tilde{s}$ vary, and the $W$-equivariance holds, since it does so fiber-by-fiber. In the general case, the $\mathcal{C}$-bundle $P^{\mathcal{C}}$ on $X$ together with the inclusion of $\mathcal{C}$ into the universal Borel bundle $\mathcal{B}$ over $\tilde{S}$ induce on $\tilde{S} \times_{S} X$ a $\mathcal{B}$-bundle, hence a quotient $T$-bundle. Our morphism $v$ is the classifying map for this bundle.

(3) Above $\tilde{S}$ we have a bundle of Borels; the $T$-bundle is associated to it as above. We will discuss the shifted $W$-equivariance below.

The main point of this note is that a regularized $G$-bundle can be reconstructed from its spectral data. To see this, it will be convenient to introduce an intermediate object, the principal $G$-Higgs bundle, cf. [2]. This is simply a pair $\left(P_{S}, \mathcal{C}\right)$ consisting of a principal $G$-bundle $P_{S}$ on $S$ together with a family $\mathcal{C}$ of regular centralizers in $\mathrm{Ad}_{\mathrm{S}}$. (To avoid worrying about how the different types of centralizers fit together, we can instead consider the vector subbundle $\underline{\mathbf{c}}$ of regular centralizers in the bundle ad $P_{S}$ of Lie algebras. This , of course, is equivalent data.) Now as above, to a principal $G$-bundle $\left(P_{S}, \mathcal{C}\right)$ corresponds an abstract cameral cover $\tilde{S} \rightarrow S$ and a homomorphism $\ell: \Lambda \rightarrow$ Pic S. (The constructions of items (1) and (3) above used only the available data on $S$.) As $W$ acts on both sides, we can consider the subgroup of $W$-equivariant homomorphisms. In [2] this was called the distinguished Prym of $\tilde{S} / S$. In case $S, \tilde{S}$ are non-singular, this is a finite group times an abelian subvariety of $\operatorname{Hom}(\Lambda, \operatorname{Pic}(\tilde{\mathrm{S}}))$.

The main observation in [2] is that the family of principal Higgs bundles with a given cameral cover $\tilde{S}$ is, if non-empty, parametrized by a translate of the distinguished Prym. The exact point by which we need to translate will not be crucial for us here. It is described in sections 5.2 and 5.3 of [2] as the sum of a cohomological shift term depending only on the group $G$, and further twist terms coming from the fixed divisors for the action of $W$ on $\tilde{S}$. An analogous and more familiar situation applies in case $G=S L(n)$ when we replace the cameral cover by the degree- $n$ spectral cover $\pi: \bar{S} \rightarrow S$ ( $\bar{S}$ is the image under $v^{\prime}$ of $\lambda_{1} \times \tilde{S}$, where $\lambda_{1}$ is the first fundamental weight.) The twisting along the ramification then corresponds to the relative Todd class (=half the ramification) which enters into the Grothendieck-Riemann-Roch formula: In order for $\pi_{*}(L)$ to have determinant $0, c_{1}(L)$ needs to be the class of half the ramification.

(The original purpose of [2] was to describe the fibers of the Hitchin map, from moduli of $K$-valued Higgs bundles on a variety $S$, to $K$-valued spectral data. Here $K$ can be the canonical bundle of $S$, as in Hitchin's original work [11], but it can also be an arbitrary line bundle, or (with some additional symmetry conditions imposed) even a vector bundle on $S$ as in Simpson's works. It turned out to be convenient to separate the problem into considerations of "eigenvectors" and "eigenvalues": we introduce the somewhat abstract "principal G-Higgs bundles" and show that they correspond to abstract spectral data (this is the eigenvector aspect); the $K$-valued versions are then recovered by adding a $K$-valued "Higgs field" $\phi$, a section of $\operatorname{ad}\left(P_{S}\right) \otimes K$, on the one side, and an "eigenvalue map" $v: \tilde{S} \rightarrow K$ on the other. This latter map is of course analogous to our datum (2). We are thus led to think of a $G$-bundle on an elliptic fibration as a sort of Higgs bundle on the base taking values in the fibration instead of in a line (or vector) bundle.)

Returning to our situation, from the spectral data (1)-(3) we thus retrieve the principal $G$-Higgs bundle $\left(P_{S}, \mathcal{C}\right)$ together with a morphism $v$ from $\tilde{S}$ (which is determined by $\left(P_{S}, \mathcal{C}\right)$ ) to $\mathcal{M}_{X / S}^{T}$, commuting with the projections to $S$. It remains to recover the original regularized $G$-bundle $\left\{P, c, P^{\mathcal{C}}\right\}$ (on $X$ ) from this data. This goes 
as follows: the situation is essentially rigid, so we can reduce to the case that $S$ is a point, i.e. to bundles on one elliptic curve. For a given regular centralizer $C$, this then reduces to the straightforward verification that the canonical map:

$$
\mathcal{M}_{E}^{C} \rightarrow \operatorname{Mor}_{\mathrm{W}}\left((\mathrm{G} / \mathrm{B})^{\mathrm{C}}, \mathcal{M}_{\mathrm{E}}^{\mathrm{T}}\right)
$$

is an isomorphism. Here $(G / B)^{C}$ is the subscheme of $G / B$ parametrizing Borels through $C$ (it is finite of length equal to the cardinality of $W$, and is not reduced except when $C$ is a torus), and Morw is the (group of) $W$-equivariant morphisms.

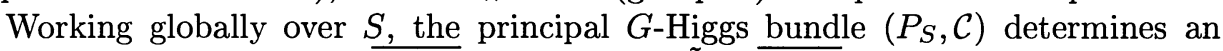
("eigenvector") map $c: S \rightarrow \overline{P_{S} / N}$ and its lift $\tilde{c}: \tilde{S} \rightarrow \overline{P_{S} / T}$, which together with the "eigenvalue" map $v: \tilde{S} \rightarrow \mathcal{M}_{X / S}^{T}$ sends $\tilde{S}$ to the fiber product $\overline{P_{S} / T} \times_{S} \mathcal{M}_{X / S}^{T}$. This map is $W$-equivariant, so it descends to give a section $\bar{\alpha}: S \rightarrow \overline{\mathcal{U}}_{X / S}^{P_{S}}:=\left(\overline{P_{S} / T} \times_{S}\right.$ $\left.\mathcal{M}_{X / S}^{T}\right) / W$. This last object is a version of our previous $\overline{\mathcal{U}}_{X / S}$ which is twisted by the bundle $P_{S}$ : it is isomorphic to $\overline{\mathcal{U}}_{X / S}$ over any open subset of $S$ over which $P_{S}$ is trivial. Likewise, we have the twisted version $\mathcal{U}_{X / S}^{P_{S}}$ of $\mathcal{U}_{X / S}$. Now, the data needed to lift our section $\bar{\alpha}$ to a section $\alpha$ of $\mathcal{U}_{X / S}^{P_{S}}$ is precisely given by $v: \tilde{S} \rightarrow \mathcal{M}_{X / S}^{T}$. (In particular, existence of $v$ implies that the image of $\bar{\alpha}$ is contained in $\overline{\mathcal{U}}_{X / S}{ }^{\prime}$.) Now on $\mathcal{U}_{X / S} \times_{S} X$ we have the universal $\mathcal{C}$-bundle trivialized along $S$. Twisting by $P_{S}$, we get a universal $\mathcal{C}$-bundle on $\mathcal{U}_{X / S}^{P_{S}} \times_{S} X$, but instead of a trivialization we now get an identification of its restriction to $S$ with the universal $\mathcal{C}$-subbundle of $P_{S}$. Pulling back via the section $\alpha$ to $X=S \times{ }_{S} X$ gives a $\mathcal{C}$-bundle $P^{\mathcal{C}}$, whose associated $G$-bundle is the original $P$.

REMARK 1: We have emphasized the cameral covers, since they are, in our view, the most basic objects in the picture. But in order to parametrize entire components of the moduli space of $G$-bundles on $X$, it is necessary to allow the cover to vary. For this purpose, it is more convenient to consider instead the universal spectral covers. These are all obtained by pulling back one object, the $W$-cover $\mathcal{M}_{X / S}^{T} \rightarrow \mathcal{M}_{X / S}^{G}$, by arbitrary sections $S \rightarrow \mathcal{M}_{X / S}^{G}$. As we saw in the previous section, $\mathcal{M}_{X / S}^{G}$ is, for most groups, a bundle of weighted projective spaces over $\mathrm{S}$. So in a connected component (obtained by fixing the numerical invariants of the map), these maps are specified by the (weighted projectivization of) the space of sections of an appropriate vector bundle over $S$. In "nice" situations, a generic map of this kind will determine a non-singular universal spectral cover, and therefore also a unique cameral cover.

REMARK 2: An alternative approach to reconstruction of a $G$-bundle on $X$ from spectral data might be based on application of the equivalence of [2] directly to the principal $G$-Higgs bundle $\left(P, \pi^{*} \mathcal{C}\right)$ on $X$. One then obtains a cameral cover $\tilde{X} \rightarrow X$ and a point $\ell_{X}$ in a translate of its distinguished Prym. It is interesting to compare this to the spectral data $\left(\tilde{S}, v, \ell_{S}\right)$ which we used above. The cover $\tilde{X} \rightarrow X$ is clearly the pullback via $\pi$ of $\tilde{S} \rightarrow S$, but $\pi^{*} \ell_{S}$ is not the same as $\ell_{X}$ : the former is trivial along fibers of $\pi$, while the behavior of the latter along fibers is equivalent to the additional datum $v: \tilde{S} \rightarrow \mathcal{M}_{X / S}^{T}$. Our original approach has a clear advantage over this alternative in situations where $X$ is interesting (say a $K 3$ or $C Y_{3}$ ) while $S$ is a much simpler object $\left(\mathbf{P}^{1}, \mathbf{F}_{n}\right)$.

\section{REFERENCES}

[1] C. VAFA, Evidence for F-theory, Nucl. Phys., B469(1996), 403, HepTh 9602022; D. MorRI- 
SON AND C. VAFA, Compactifications of F-theory on Calabi-Yau threefolds: I, II, HepTh 9602114, HepTh 9603161 .

[2] R. Donagi, Spectral covers, in: Current topics in complex algebraic geometry, Math. Sci. Res. Inst. Publ., 28 (Berkeley, CA 1992/92), 65-86, AlgGeom 9505009.

[3] E. Looljenga, Root systems and elliptic curves, Inv. Math., 38(1976), 17-32; and Invariant Theory for generalized root systems, Inv. Math., 61(1980), 1-32.

[4] J. Bernstein AND O.V.ShVARTSMAn, Chevalleys theorem for complex crystallographic Coxeter groups, Funct. Anal. Appl., 12(1978), 308-310.

[5] K. Wirthmuller, Root systems and Jacobi forms, Comp. Math., 82(1992), 293-354.

[6] R. Friedman, J. Morgan and E. Witten, Vector bundles and F-theory, HepTh 9701162.

[7] M. Bershadsky, A. Johansen, T. Pantev and V. Sadov, On four-dimensional compactifications of F-theory, HepTh 9701165.

[8] V. Baranovsky AND V. GinzBurg, Conjugacy classes in loop groups and G-bundles on elliptic curves, AlgGeom 9607008.

[9] M. AтIYAH, Vector bundles over an elliptic curve, Proc. LMS, VII(1957), 414-452.

[10] K. SAITo, Extended affine root systems, Publ. RIMS Kyoto, I: 21(1985), 75-179; and II: 26(1990), 15-78.

[11] N. Hitchin, Stable bundles and integrable systems, Duke Math. J., 54(1987), 91-114. 